Quali valori e quale bellezza per l'architettura italiana nell'epoca della comunicazione globale? Questa è la domanda che fa da sfondo al volume. L'intenzione è quella di scomporre strumentalmente la complessità dell'argomentazione attraverso il ricorso a questi due termini, ambigui, sfuggenti e per certi versi difficilmente separabili l'uno dall'altro: la bellezza, infatti, non è forse un valore in sé stessa? E nell'evidenza di un valore di riferimento non si realizza, implicitamente, l'epifania del bello nell'opera?

I contributi raccolti in questo libro sono emblematici dei differenti modi di intendere il rapporto tra riflessione teorica e progetto e rappresentativi di come nella ricerca si possano praticare strade anche distinte, tuttavia assolutamente legittime per il raggiungimento degli esiti. Essi dimostrano anche come l'articolazione plurale delle posizioni, e quindi delle tecniche di ricerca, sia una ricchezza fondamentale per una comunità scientifica che si occupa di architettura. Perché sia chiaro che possiamo farci ancora latori di un messaggio che, su entrambe le parole chiave, appartiene da un lato alla tradizione italiana (intesa come l'insieme degli studi che l'hanno caratterizzata e delle opere che ne sono testimonianza) e dall'altro al futuro, per la costruzione del quale lavoriamo nella formazione, nella ricerca e nel progetto.

Con un testo di Edoardo Albinati.

Orazio Carpenzano, è professore ordinario di Progettazione Architettonica e Urbana presso la Facoltà di Architettura della Sapienza Università di Roma. Dirige il Dipartimento di Architettura e Progetto, il portale Archidiap e le collane DiAP PRINT. Suoi progetti e scritti appaiono su pubblicazioni e riviste nazionali e internazionali.

Dina Nencini, è professore associato in Composizione Architettonica e Urbana presso la Facoltà di Architettura della Sapienza Università di Roma. Attualmente è coordinatrice del Dottorato di ricerca in Architettura e Costruzione DRACo. Tra le sue pubblicazioni La piazza. Ragioni e significati nell'architettura italiana (2012), Past Forward. Chongqing, Shanghai and other Italian stories (2017) e Libro bianco libro nero. Le ragioni dell'invenzione (2018).

Manuela Raitano, è ricercatore presso la Sapienza Università di Roma. È docente presso il Master PARES e presso il Dottorato di ricerca in Architettura e Costruzione DRACo. Ė autrice di Dentro e fuori la crisi. Percorsi di architettura italiana del secondo Novecento (Librìa, 20I2). Per la collana DiAP PRINT ha curato, con Pisana Posocco, il volume La seconda vita degli edifici. Riflessioni e progetti (2016).

ISBN 978-88-229-0187-3

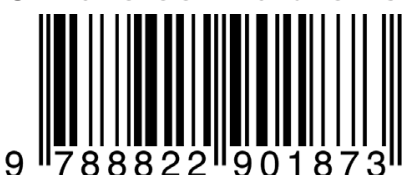

Architettura in Italia I valori e la bellezza

a cura di

Orazio Carpenzano Manuela Raitano

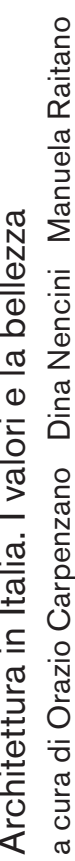

Dina Nencini

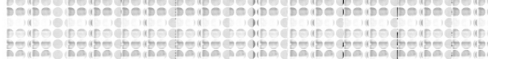

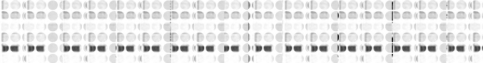

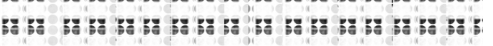

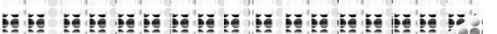

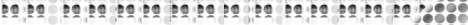
$\ldots$

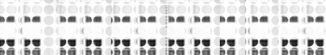

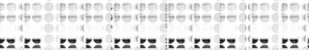

at o.

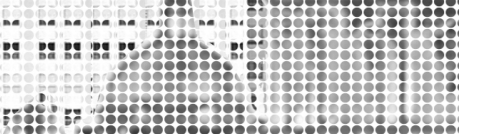

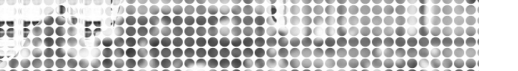

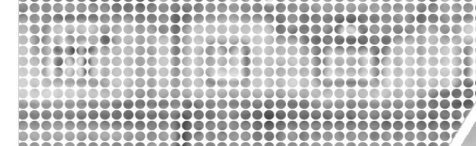


DIAP PRINT / TEORIE 14 
Architettura in Italia
I valori e la bellezza

A cura di Orazio Carpenzano,

Dina Nencini, Manuela Raitano

Quodlibet 
DiAP Dipartimento di Architettura e Progetto

Direttore Orazio Carpenzano

Sapienza Università di Roma

DiAP PRINT / TEORIE

Collana a cura del

Gruppo Comunicazione del DiAP

Coordinatore Cristina Imbroglini

COMITATO SCIENTIFICO

Carmen Andriani

Roberta Amirante

Jordi Bellmunt

Renato Bocchi

Giovanni Corbellini

Giovanni Durbiano

Carlo Gasparrini

Sara Marini

Luca Molinari

Alessandra Muntoni

Franco Purini

Joseph Rykwert

Andrea Sciascia

Zeila Tesoriere

Ilaria Valente

Herman van Bergeijk

Franco Zagari

Ogni volume della collana è sottoposto alla revisione di referees esterni al Dipartimento di Architettura e Progetto scelti tra i componenti del Comitato Scientifico. (c) 2018

Quodlibet srl

via Giuseppe e Bartolomeo Mozzi, 23

Macerata

www.quodlibet.it

PRIMA EDIZIONE

aprile 2018

ISBN

978-88-229-0187-3

IN COPERTINA

Elaborazione grafica di Mariangela Ludovica Santarsiero 
Indice

9 Nota dei curatori. Le ragioni di un incontro

I3 Preludio

Orazio Carpenzano

25 Valori e bellezza. Contemporanee fertili inquietudini e necessarie azioni

Dina Nencini

33 Via il piombo dalle ali! Manuela Raitano

Parte prima. Di bellezze e di valori

45 Il "politeismo dei valori” dell'architettura italiana Marco Biraghi

53 Fuori dalla crisi: rospi, bellezze e il sapere dell'architetto Roberta Amirante

63 La bellezza è un fraintendimento Carmen Andriani

77 Salvare la bellezza. L'architettura italiana al bivio Luca Molinari

87 La bellezza come disvelamento della natura delle cose Carlo Moccia 
INDICE

95 L'architettura come arte positiva

Franco Purini

I07 L'architettura è solo per una stirpe felice?

Anna Irene Del Monaco

I 2 I Bellezza e commisurazione

Mariangela Ludovica Santarsiero

I 29 L'esperienza complessa dei corpi tra mente e ambiente. Architettura abito del corpo

Giovanni Rocco Cellini

I37 Ordine e disordine, armonie e dissonanze

Alessandra Capanna

I47 Bellezza o dello sguardo critico

Antonino Saggio

I 55 Legami ambigui e necessari tra bellezza e valori

Luca Porqueddu

I65 La bellezza come diritto e come espressione di dignità civile Piero Ostilio Rossi

I77 Il paesaggio tra valori e bellezza Alessandra Capuano

Parte seconda. Architetture in Italia

I 89 Dieci note per una bellezza possibile

Michele Beccu

20I L'indicibilità della bellezza e l'appropriatezza della costruzione Bruno Messina

2 I I Architettura e tecnica di guerriglia

Cherubino Gambardella 
2 I 7 Le idee e il progetto

Vittorio Pizzigoni

225 Lo spazio pubblico come valore. Figure della bellezza negli spazi della mobilità

Filippo Lambertucci

235 La ricerca dell'aura perduta

Aldo Aymonino

Parte terza. Letture

245 Archeologia del bello

Anna Giovannelli

255 Tra valore d'uso e funzione

Lelio Di Loreto

265 Valori mutevoli nelle case di vacanza di Gio Ponti

Pisana Posocco

277 La bellezza ha uno spirito interiore

Simona Ottieri

283 Valori e bellezze negli interventi di piccola scala

Gianpaola Spirito

29 I Il diritto al brutto di Lina Bo Bardi e la bellezza dell'architettura popolare

Alessandra Criconia

303 Effetti collaterali

Edoardo Albinati 


\section{Valori mutevoli nelle case di vacanza di Gio Ponti}

Pisana Posocco

\section{I valori di "Domus"}

Alcuni articoli di Gio Ponti pubblicati sulla rivista "Domus" sembrano avere, più che uno scopo divulgativo, l'intento di illustrare case ed architetture in modo tale da renderle desiderabili e determinarne così una domanda economica ed un'aspirazione sociale.

"Domus" fu fondata da Ponti nel i928. Gianni Mazzocchi, l'editore, pensava ad una rivista per signore che affrontasse vari temi, per lo più legati alla casa. Ponti, invece, aveva in mente una testata per amanti delle arti e dell'architettura, donne e uomini, architetti, artisti, e più in generale orientata verso una fascia sociale - la borghesia - in grado di apprezzare e godere di queste cose. Una rivista di stampo interdisciplinare che affrontasse temi di arte, artigianato, arredamento, architettura, sarebbe stata infatti uno strumento per la diffusione di un determinato gusto, avrebbe potuto suggerire un modo di vivere moderno ma all'italiana. "Domus" presentò, come normalmente fanno le riviste femminili di moda con gli abiti per l'estate, una serie di numeri monografici dedicati alle case al mare. Questi numeri uscirono puntualmente nei mesi estivi: il I3 8 nel giugno I939, il I40 nell'agosto I939 il I 52 nel successivo agosto I940. Ci furono anche una serie di numeri speciali dedicati alle case in campagna (I36, aprile I939) e quelle in montagna (I4I, settembre I939). In generale molte sono le case di vacanza inserite nei numeri di quel periodo: si potrebbe dire che in quegli anni questo fu un tema che impegnò Gio Ponti in modo intenso e costante.

Nella rivista "Domus" le pagine che illustrano raffinati e pre- 
gevoli oggetti d'arredo, che si potrebbero ben dire di lusso, si trovano di fianco ad altre, in cui sono presentate case di vacanza dall'aspetto semplice, quasi dimesso, con spazi ed attrezzature assai differenti dalle consuetudini borghesi allora diffuse. Con la stessa tecnica che veniva utilizzata per la pubblicità subliminale, Ponti inserisce tra le pagine di una rivista che racconta oggetti e pratiche borghesi alcuni piccoli edifici per vacanza (al mare, in campagna ed in montagna) in cui si svolgono vite ed hanno luogo rituali diversi da quelli che la consuetudine ha riconosciuto idonei per la "buona società".

Nei primi decenni del secolo i valori a cui si impronta la vacanza sono in rapido mutamento: da villeggiatura per pochi, la vacanza si trasformerà in svago per molti e poi per tutti (ciò succederà pienamente solo nel secondo dopoguerra). Gio Ponti lavora per la società borghese, o vi fa più o meno consciamente riferimento. Ciò nonostante, un po' alla volta, i valori che riconosce e su cui modella le case sono sempre meno quelli della celebrazione e della rappresentazione del ruolo sociale. Sembra piuttosto ripiegare su valori più intimi ed umani. Questo mutare dei valori determinerà, un po' alla volta, il cambiamento stilistico delle sue case di vacanza. Lo scopo dell'architettura di Ponti, soprattutto quella per il tempo libero, sembrerebbe vieppiù riassumersi nella volontà di modellare spazi che permettano di essere felici, o addirittura che inducano ad esserlo. In particolare la felicità, durante una vacanza in riva al mare, è tutta nella gioia di vivere, nel ritrovare il rapporto - intellettuale e fisico - con la natura e con il paesaggio. Nelle parole di Ponti sembra riecheggiare una lode latina per l'otium come modello di vita. Le sue case di vacanza esibiscono una bellezza antica, un'arcaicità che diventa un'alternativa italica e mediterranea al moderno internazionale.

\section{Le case di vacanza}

La prima casa "da week-end" che Gio Ponti progetta e pubblica su "Domus" 'è del I928: è un piccolo edificio con portico

${ }^{1}$ Cfr. "Domus", aprile I928, p. 26. 
e colonne, di vago sapore palladiano, che molto deve alla villa de L'Ange volant realizzata a Garche (Parigi) assieme a Tomaso Buzzi ed Emilio Lanza nel 1926.

Successivamente Ponti elabora, sempre con Lancia, una casa per la vacanza, per conto della Rinascente e della società De Angeli Frua, da presentare al concorso indetto dalla IV Triennale di Monza ( 9930), della quale Ponti era, assieme ad Alpago Novello e Sironi, membro del comitato organizzativo. Si tratta di una villa di piccole dimensioni, il cui aspetto e le cui decorazioni sono di stampo neoclassico. Per contestualizzare l'opera e le scelte di Ponti è utile ricordare che tra le architetture realizzate per quell'occasione c'era la famosa "casa elettrica” del Gruppo $7^{2}$ - un simbolo della modernità, anche in virtù dell'introduzione dell'elettrificazione domestica -, da molti ritenuta il primo esempio di architettura razionalista in Italia. Molta è la distanza tra le due architetture.

La casa per le vacanze di Ponti e Lanza sarà pubblicata sul numero di settembre di "Domus" 3 ; Ponti dichiara sin da subito che questo tema (per il quale utilizzerà anche i termini di bungalow o week-end house) lo seduce e, a suo parere, interessa enormemente il pubblico. All'esposizione di Monza ${ }^{4}$ ce n'erano più di una. I nomi dati erano vari e spesso erano indicate come "case del dopolavorista", qual è in genere il tipo di casa di vacanza che si va diffondendo: non più la villa di villeggiatura delle classi dominanti, che riassumono in quella tipologia la dimora legata alle proprietà terriere da cui derivano le loro rendite ed il luogo di svago alternativo allo stare in città e al lavoro. Alla Triennale del I930 era stata costruita una "casa del dopolavorista" progettata dall'arch. Luisa Lovarini: era un progetto voluto e finanziato dall'Opera Nazionale Dopolavoro che doveva essere il manifesto della politica sociale del regime e del suo concetto di assistenzialismo alle masse lavoratrici; di questa Ponti dice: "potrebbe

${ }^{2}$ In particolare il progetto edilizio della casa-padiglione è da ricondursi a Luigi Figini e Gino Pollini; contribuirono al disegno degli interni Piero Bottoni, Guido Frette e Adalberto Libera.

3 "Domus", 33, 1930, pp. 19-25.

${ }_{4}$ Cfr. Catalogo ufficiale della IV Esposizione Triennale Internazionale delle Arti Decorative e Industriali Moderne, Ceschina, Milano 1930. 
essa pure essere adatta come casa di campagna di questo genere (riferendosi a quella progettata da lui e da Lanza) meglio forse che rappresentare l'abitazione tipica del dopolavorista. Le nuocerebbero però in quella destinazione i suoi studioli e salotti e verandine di borghese memoria"s.

Nel descrivere quella da loro progettata, Ponti indica come tema fondamentale il salotto e richiama il termine inglese di living-room per indicare che si tratta del posto "dove si lavora, si legge, si mangia, si chiacchiera, si vive insomma" ${ }^{6}$. È questo il cuore della casa per la vacanza. Alla descrizione della funzione seguono indicazioni figurative: "nel nostro caso la sala di soggiorno occupa la facciata e si apre su di essa con quattro finestre. Il taglio di questa architettura è volutamente nostrano, e la costruzione s'è voluto che fosse in mattoni" ". È da osservarsi che lo stile adottato, lo stile "nostrano", è una sorta di classicismo: la facciata è caratterizzata da quattro aperture, due finestre e due porte-finestre, che sono decorate da timpani e lunette di semplice fattura, ma che costituiscono l'elemento caratterizzante e decorativo principale. La maniera architettonica diffusa all'indomani della prima guerra mondiale era un classicismo di tradizione, modo radicato e consuetudine consolidata in Italia, in particolare presso le classi borghesi. Per Ponti il classico era il modo per esprimere l'identità italiana, era una maniera che permetteva di avere una continuità con la tradizione, ma al contempo, secondo la lezione del novecentismo milanese, di essere moderni. Nei suoi scritti su "Domus" traspare il rispetto che aveva per la tradizione architettonica ed artistica italiana: egli parla con orgoglio tanto delle forme classiche così come dei materiali della tradizione ${ }^{8}$.

La sua lettura del classico però, forse proprio perché era passata attraverso le arti minori e perché proprio lui ne aveva fatto una riduzione adatta ad essere utilizzata per il disegno della ce-

${ }^{5}$ Ivi, p. I9.

${ }^{6}$ Ibid.

7 Ibid.

${ }^{8} \mathrm{~A}$ tal riguardo è interessante e molto ben documentato il testo di M. Lamonaca, Tradition as Transformation: Gio Ponti's Program for the Modern Italian Home, I928-1933, "Studies in the Decorative Arts", v, I, I997, pp. 52-82. 
ramica e dell'arredamento, perderà la fissità della tradizione e si farà scherzosa, ironica, si potrebbe dire "nuova" e "fresca". In tal senso il decorativismo neoclassico si trasforma in un uso moderno di materiali della tradizione, come ad esempio la ceramica, che poi resterà una cifra dell'opera di Ponti.

Per tornare alle case per la Triennale di Monza, bisogna notare che la descrizione si concludeva però in modo tale che già si aprivano seminali riflessioni per nuovi sviluppi: “[...] È però da notare, quando si parla di questo tipo di abitazioni di campagna, che non si tratta di una casetta economica: questo tipo di costruzione appartiene anzi a quelle manifestazioni caratteristiche di una classe educata al gusto ed al comfort".

\section{Il conforto dell'architettura}

La riflessione sul comfort sarà un elemento rilevante per Ponti nella modifica dei valori attribuiti alla casa di vacanza: non più luogo di celebrazioni sociali ma luogo dell'otium. E quindi la priorità, nel momento di elaborazione degli spazi di una dimora, passa dalla definizione stilistica dell'immagine al plasmare spazi per la vita e il riposo fisico ed intellettuale.

Già nel I928, nel primo numero di "Domus", scriveva un famoso testo sulla Antica casa all' italiana ${ }^{\mathrm{Io}}$, che così inizia: "La casa all'italiana non è il rifugio, imbottito e guarnito, degli abitatori contro la durezza del clima, come è delle abitazioni d'oltre alpe ove la vita cerca, per lunghi mesi, riparo dalla natura inclemente; la casa all'italiana è come il luogo scelto da noi per godere in vita nostra, con lieta possessione, le bellezze che le nostre terre e i nostri cieli ci regalano in lunghe stagioni. Nell'antica casa italiana non vi è grande distinzione d'Architettura fra interno ed esterno"; e, dopo aver illustrato il rapporto tra il dentro ed il fuori della dimora italica, affronta il tema del comfort: "Il suo disegno non discende dalle sole esigenze materiali del vivere. Il cosiddetto

9 Ponti diresse "Domus" dal I928 al I94 I e poi ancora dal I948 al I979.

10 G. Ponti, Antica casa all'italiana, "Domus", I, I928; poi in Id., Amate l'architettura, Vitali e Ghianda, Genova I957, p. I06. 
1. Gio Ponti, Emilio Lancia, Progetto di una casa di campagna, 1930.

2. Gio Ponti, Emilio Lancia, La Casa delle Vacanze, 1930.

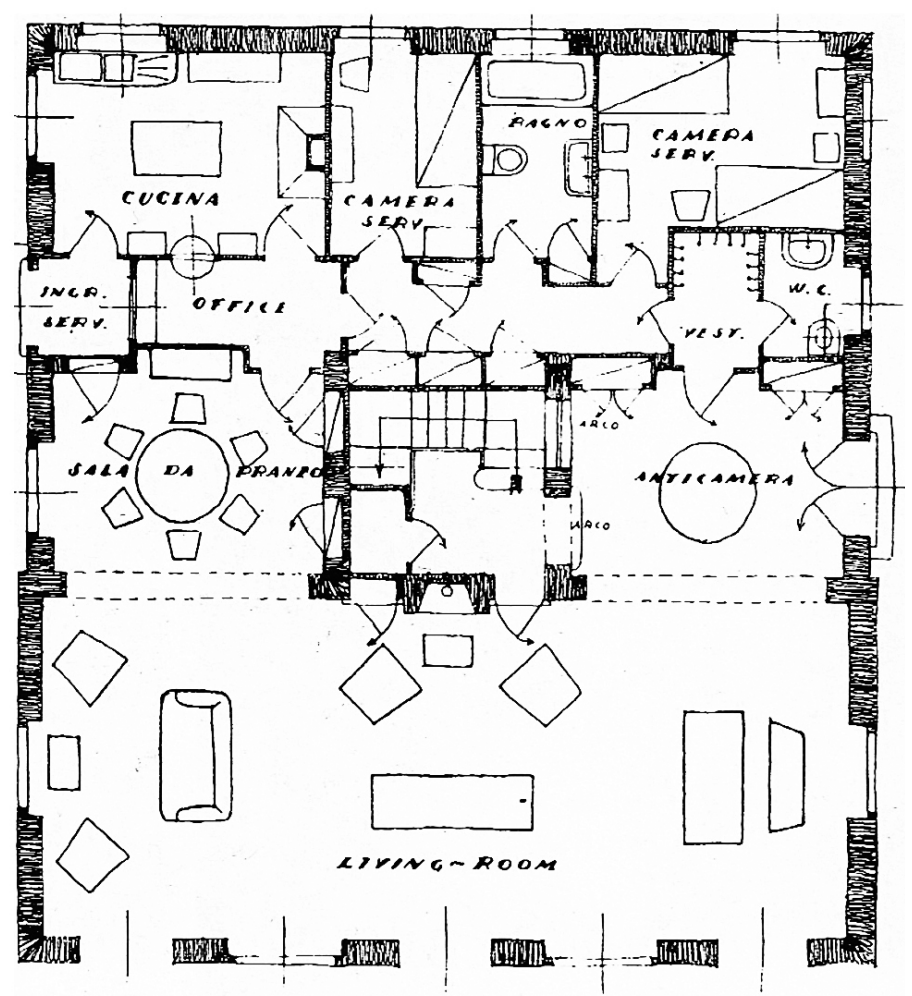

Piano superiore

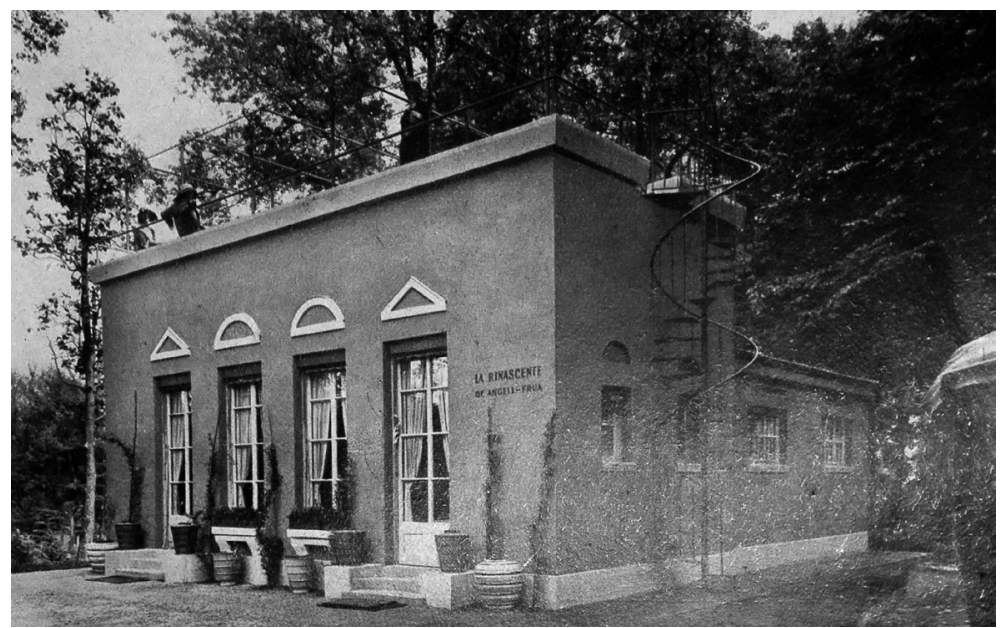




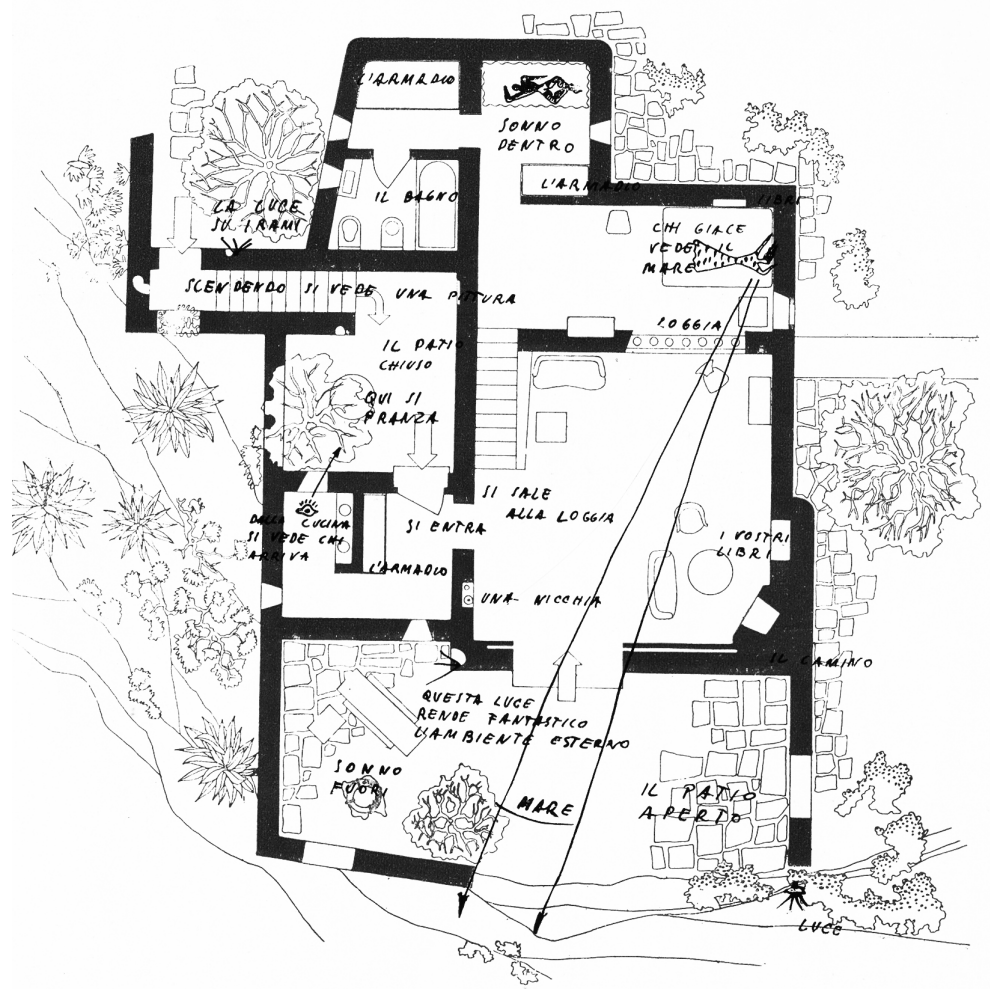

3-4. Gio Ponti, Una casetta al mare, "Domus", 140, 1939.

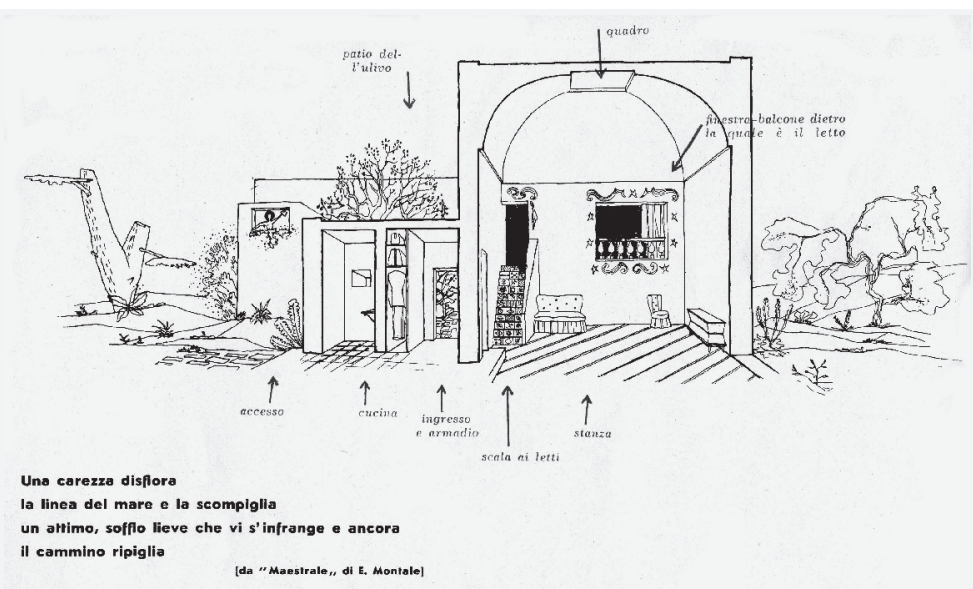


'comfort' non è nella casa all'italiana solo nella rispondenza delle cose alla necessità, ai bisogni, ai comodi della nostra vita ed alla organizzazione dei servizi. Codesto suo 'comfort' è in qualcosa di superiore: è nel darci con l'architettura una misura per i nostri stessi pensieri, nel darci con la sua semplicità una salute per i nostri costumi, nel darci con la sua larga accoglienza il senso di una vita confidente e numerosa, ed infine, per quel suo facile, lieto e ornato aprirsi fuori e comunicare con la natura, è nell'invito che la casa all'italiana offre al nostro spirito di ricrearsi in riposanti visioni di pace, nella solare natura; nel che consiste, nel pieno senso della parola italiana, il conforto".

Emerge con forza questa idea di un ideale umanistico e non meccanico $^{\text {II }}$, quasi a segnare la distanza dai valori dell'International Style e a dichiarare propri quei valori che sostiene e promulga attraverso le sue case di vacanza mediterranee.

Sicuramente la casa moderna italiana fu il tema principale su cui Ponti si cimentò, si concentrò, sperimentò e rifletté per quasi tutta la sua vita. In particolare nel periodo tra il 1928 e gli inizi degli anni '3o. Le variazioni su questo tema riflettono le trasformazioni del suo pensiero, il variare delle sue posizioni, come pure delle caratteristiche delle sue architetture.

Sul finire degli anni ' 30 e a cavallo con gli inizi del decennio successivo, su "Domus" vengono pubblicate molte case di vacanza, soprattutto "ville mediterranee"; in particolare molti sono i progetti di Ponti presentati con bellissimi disegni chiosati da commenti che esplicitano le scelte fatte per ciascun ambiente, per ciascuna funzione, per ciascun momento rilevante della vita. Questi progetti sembrano dar forma alla Casa all'italiana, che Ponti aveva teorizzato nel 1928 e letterariamente e teoricamente descritto in un testo apparso su "Domus".

${ }^{11}$ Cfr. G. Ponti, Una abitazione dimostrativa alla IV Triennale, "Domus", ı03, I936: "la vita contemporanea, moderna come la concepiamo ha per postulato, la presenza di uno spirito umano lieto, puro, senza istinti borghesemente mortificati, e pratico ma nutrito di tutte le attualità spirituali della vita d'oggi. Ideale umanistico e non meccanico: nel senso di un umanesimo non antimeccanico ma che ha assimilato la macchina e i suoi derivati”. 


\section{Gio Ponti e Bernard Rudofsky: la casa mediterranea}

Nel periodo tra il I937 e il I938 Gio Ponti frequenta Bernard Rudofsky. È il momento in cui le sue posizioni sulla “casa all'italiana" si modificano.

Rudofsky aveva a lungo, forse per una vita intera, ragionato attorno ai temi della mediterraneità e dell'architettura popolare, anonima, contadina, spontanea; di lui Andrea Bocco Guarneri dirà che è stato "uno degli autori che più hanno influenzato sia il cambiamento di attribuzione di valore nei loro ${ }^{\text {I2 }}$ confronti, sia l'innesto della pluralità (diversità culturale come ricchezza in sé) e dei valori locali nella cultura architettonica dopo la tabula rasa modernista" ${ }^{13}$.

"Il Mediterraneo insegnò a Rudofsky, Rudofsky a me" I4, questo dirà Ponti per spiegare la sua relazione ed il suo debito; e fu così che le sue case (e più in generale i progetti: è infatti necessario ricomprendere la proposta per l'Albergo nel Bosco che elaborò con Rudofsky) diverranno anti-classiche: faranno loro il modo antico di vivere l'otium e si conformeranno ad esso ma perderanno quasi totalmente le fattezze neoclassiche per farsi "povere" e "mimetiche" rispetto al paesaggio mediterraneo. Non saranno né l'uno né l'altro. Il mito del Mediterraneo si trasformerà, non sarà più la culla del classico, ma l'Arcadia. $\mathrm{Al}$ cambiamento dei valori segue un mutato canone di bellezza. Le case non parlano più la lingua dei timpani e delle colonne, ma utilizzano etimi rustici e popolari per dare continuità al tema della vita arcaica e bucolica. Come scriverà in un altro pezzo rimasto famoso, "non ci vuole un nuovo modo di costruire, ci vuole un nuovo modo di vivere" ${ }^{15}$.

Questo suo ragionamento in astratto, sui massimi sistemi, ha una ricaduta immediata e chiara e si ritrova, con la stessa poten-

${ }^{12}$ Il riferimento è all'architettura popolare, anonima, contadina, spontanea.

${ }^{13}$ A. Bocco Guarneri, Appunti sul rapporto tra Bernard Rudofsky e il Mediterraneo, "Firenze Architettura", I, 20 I I, p. 78.

${ }^{14}$ G. Ponti, James S. Plaut, Aria d'Italia. Espressione di Gio Ponti, Daria Guarnati, Milano I954, p. 25.

${ }^{15} \mathrm{G}$. Ponti, Non ci vuole un nuovo modo di costruire ci vuole un nuovo modo di vivere, "Domus", I23, I938, pp. 6-I 5. 
za, in specifici progetti elaborati per specifiche situazioni. Così commenta infatti una casa sulla costa ligure: "Batto e ribatto sulla necessità assoluta di semplificare il gusto delle costruzioni delle nostre Riviere. Tutte le vecchie case lungo le marine e quelle coloniche fra gli ulivi o i pini dei colli in riva al mare, sono belle: tutte le pretenziose ville (con la vanerella torre!) fatte dopo, dalla infausta classe dei villeggianti, sono un'ira di Dio, che deturpa le nostre spiagge e ne diminuisce dannosissimamente quelle attrattive turistiche alle quali dobbiamo tenere tanto. Oggi gli architetti hanno una sensibilità assolutamente superiore e fanno le cose davvero 'a proposito"' "'.

\section{L'Anonimo}

Proprio in quegli anni, subito dopo la gran messe di case per la vacanza, Ponti ospita sulle pagine della rivista una serie di testi di E.N. Rogers che si intitolano Confessioni di un Anonimo del $\mathrm{XX}$ secolo $^{\mathrm{I7}}$.

Pur nei suoi modi pacati ed apprezzati dal mondo borghese, Ponti sembra registrare più di altri la grande frattura nell'apparente continuità che ha contraddistinto l'architettura moderna del xx secolo in Italia. Giovanni Leoni, in un saggio che affronta e illustra il tema dell' "anonimo" ${ }^{\mathrm{I} 8}$ e chiarisce la sua importanza nella cultura architettonica italiana a cavallo della metà del seco28-29.

${ }^{16}$ G. Ponti, Una nuova casetta a Quinto sul mare, "Domus", I 54, I940, pp.

${ }^{17}$ I testi sono ricompresi in due annate: I940 e I94I. In part. cfr. "Domus": I 58, Presentazione dell'Anonimo (p. 45); I59, Le Coordinate dell'Anonimo (p. 67); i60, L'Anonimo e la folla (p. 59); I6I, I confini dell'Anonimo (p. 69); I62, L'Anonimo nel tempo (p. 69); I64, I sogni dell'Anonimo (p. 3 I); I67, Responsabilità dell'Anonimo (p. I7); I70, La personalità dell'Anonimo (p. 93); I76, La casa dell'Anonimo (p. 333).

${ }^{18} \mathrm{G}$. Leoni, L'Anonimo come tema di discontinuità nella cultura architettonica italiana tra Primo e Secondo Novecento, in C. Togliani (a cura di), Un palazzo in forma di parole. Scritti in onore di Polo Carpeggiani, Franco Angeli, Milano 2016, pp. 463-472. Il testo è stato ripreso in Anonymous as a Theme of Discontinuity in the Culture of Italian Architecture between the First and Second Halves of the 2oth Century, "HPA. History of Postwar Architecture", 0, 20I7, p. I05. 
lo, sottolinea soprattutto come abbia rappresentato un motivo di rottura e discontinuità. La riflessione non è solo sull'architettura anonima, sullo "stile anonimo" ma è soprattutto l'operare "anonimo" dell'architetto ad essere al centro delle riflessioni di Ernesto Rogers, è "accettazione del combattimento" tra la personalità e la sua scomparsa ${ }^{19}$. Queste architetture vogliono essere anonime non solo nello stile ma anche nella voluta assenza di un tratto di autorialità. È Rogers che parla, ma sono tratti che si possono individuare nelle architetture di Ponti. Quanto anonime sono le sue case di vacanza all'Isola d'Elba agli inizi degli anni '6o? Quanto è chiara la sua ricerca, per le case di vacanza, di un modo - non solo uno stile - per costruire nuovi manufatti anonimi che, se dovranno dispiegarsi lungo le coste italiche, lo facciano con il pudore di chi non vuole a tutti i costi apparire? Non è l'architetto artista che deve apparire dietro a queste opere, è l'Anonimo. Il design deve essere anonimo.

Leoni scrive che il progetto anonimo per Rogers è un luogo in cui il semplice atto dell'uomo comune si incontra e si fonde con quello del progettista anonimo. Non c'è spazio per il segno soggettivo ed autoriale. Il progetto anonimo è "l'inversione radicale del principio funzionalista e di ogni leggenda associata, l'abbandono del compito di interpretare i bisogni umani classificabili e di offrire loro una forma architettonica resa possibile per una comunità".

Queste le posizioni di Rogers, che proprio in "Domus" trovano spazio.

E per tornare alle parole di Ponti, in cui forte echeggia Rogers, così racconta un suo progetto: "Questa mia piccola costruzione è nata da un'ispirazione del luogo che si è incontrata con un mio antico desiderio di fare una casa tutta allungata sul bordo del mare e riassumere le mie idee sulle ville al mare, che vorrei tutti amassero così: semplici, murarie, luminose e, dove occorre, ombrose di portici. Io penso, convintissimo, che questa semplicità sia il raggiungimento di un lusso dello spirito

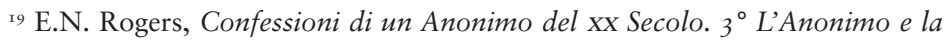
folla, "Domus", I60, I94I, p. 59. 
e che ogni aggiunta di ricchezza conduca assolutamente a un risultato inferiore" ${ }^{20}$.

Ma se qui aveva espresso un commento su di una sua opera, sembra ancora più interessate osservare come chiosa un progetto di Minoletti per una zona di ville in Liguria: "Sopra S. Margherita Ligure [...] sta sorgendo [...] un gruppo di case per vacanza, tutte, pur nella varietà delle forme e delle entità, omogenee nello spirito e nel gusto, nella volontà di rispetto di una comune armonia e nella interpretazione della parola 'vacanza'.

Le case variano soprattutto per il numero di persone che vi possono vivere, ma in tutte è costante la ricerca di una cornice adatta alla vita semplice, sana, comoda e piacevole.

Tutte le case sono o saranno costruite con materiali locali e metodi semplici di costruzione, perché ne sia limitato il costo, e le forme architettoniche siano custodite da questi metodi costruttivi in una schietta sincerità, non dissimile da quella che ispira le vecchie case rurali liguri. [...] Tutto il piccolo villaggio, se così si può chiamare, è stato pensato dall'architetto Giulio Minoletti, che ha saputo dare, coi disegni che presentiamo, un'altra prova di quanto da tempo 'Domus' afferma. L'unità di carattere stilistico e, oseremmo dire, urbanistico; il gusto moderno che ispira le piccole architetture; il loro accordo con la vita del luogo, vita testimoniata dalle esigenze del clima e dalla possibilità dei materiali e delle particolari esperienze costruttive, indicano questo complesso architettonico come una buona soluzione dei problemi che noi continuamente affacciamo all'attenzione dei nostri lettori" ${ }^{21}$.

A ben osservare, le architetture che Ponti presenta in quel periodo sono piuttosto omogenee, sono la sua versione del progetto "anonimo", la nuova maniera di fare architettura, in particolare architettura per la vacanza, il lusso dello spirito che propone e suggerisce.

${ }^{20}$ G. Ponti, Una casa al mare, "Domus", I3 8, I939, p. 34.

${ }^{21}$ G. Ponti, Una zona di ville in Liguria, "Domus", I40, I939, p. 39. 